\title{
Elevated serum levels of cathelicidin and $\beta$-defensin 2 are associated with basal cell carcinoma
}

\author{
MARTA FIJAEKOWSKA ${ }^{1}$, MAREK KOWALSKI, MATEUSZ KOZIEJ ${ }^{3}$, BOGUSEAW ANTOSZEWSKI ${ }^{1}$ \\ ${ }^{1}$ Department of Plastic, Reconstructive and Aesthetic Surgery, ${ }^{\text {nd }}$ Chair of Surgery, Medical University of Lodz, Lodz, Poland \\ ${ }^{2}$ Department of Immunology and Allergy, Medical University of Lodz, Lodz, Poland \\ ${ }^{3}$ Department of Anatomy, Jagiellonian University Medical College, Krakow, Poland
}

\begin{abstract}
Antimicrobial peptides (AMPs) are one of the primary mechanisms used by the skin in the early stages of immune defense. AMPs have a broad antibacterial activity and also show antifungal and antiviral attributes. Various studies have also shown that levels of antimicrobial peptides change with the development of neoplasia. The aim of this paper is to assess the associations between the presence of basal cell carcinoma (BCC) and the plasma concentrations of cathelicidin and $\beta$-defensins (HBD1-3). We examined 108 patients (56 women, 52 men). The BCC group consisted of 49 patients with mean age $69.8 \pm 12.3$ and the control group consisted of 59 participants with mean age $62.1 \pm 11.1$. A statistical analysis of data was performed. The median serum concentration of cathelicidin was almost 3 times higher and the median concentration of HBD-2 more than 6 times higher in BCC patients than in the control group ( $p$ $<0.001)$. The logistic regression model revealed in univariate analysis that patients who had a detected cathelicidin level above $1500 \mathrm{pg} / \mathrm{ml}$ had $9.9 \times$ higher likelihood of having BCC identified in the histopathology in comparison with the control group. In patients who had a HBD-2 level above $\sim 1.2 \mathrm{ng} / \mathrm{ml}$ the OR of having BCC identified in the histopathology was $12.6(p<0.001)$. Elevated concentrations of cathelicidin and $\beta$-defensin 2 are associated with the presence of basal cell carcinoma. Additionally, the specificity of cathelicidin and $\beta$-defensin 2 in detecting basal cell carcinoma is high. However, it should be remembered that these factors are not specific only to this condition and further studies are needed.
\end{abstract}

Key words: cathelicidin, $\beta$-defensins, basal cell carcinoma, risk.

(Cent Eur J Immunol 2021; 46 (3): 360-364)

\section{Introduction}

Antimicrobial peptides (AMPs) are one of the primary mechanisms used by the skin in the early stages of immune defense. AMPs have broad antibacterial activity and also show antifungal and antiviral attributes. Additionally, AMPs act on host cells to stimulate cytokine production, cell migration, proliferation, maturation, and extracellular matrix synthesis [1]. Later it was also discovered that AMP levels change during the development of neoplasia [2]. The family of AMPs is quite broad but mainly it includes defensins, cathelicidin, dermcidin and psoriasin [1]. The defensins are a group of small cationic peptides and are categorized in three subfamilies $-\alpha-, \beta-$, and $\gamma$-defensins - but only $\beta$-defensins are detected in human skin $[1,2]$. Cathelicidin (LL-37) is named for the conserved prosequence domain of the precursor protein that resembles the cathelin protein, originally isolated as a cathepsin L inhibitor [3].

Human $\beta$-defensins (HBDs) can be up- or down-regulated depending on the specific cancer type and its anatom- ical location [2]. Particularly human $\beta$-defensin 2 (HBD-2) has been reported to be upregulated in several cancers, including esophageal [4], lung [5, 6], cervical [7], and skin cancer (BCC and SCC) $[8,9]$, but it has been described to be downregulated in colon cancer [10]. Studies presented in the literature have shown that cathelicidin can promote malignant tumors, including cancers of the lungs, ovaries, prostate, melanoma and oral squamous cell carcinoma; and this process is primarily associated with the upregulation of EGFR and the receptor tyrosine kinase ErbB2 [11, 12]. Through the induction of the membrane-associated protein kinase, EGFRs cleave the matrix metalloproteinase (MMP), and LL-37 activates EGFR [13, 14].

Different studies have shown that defensin levels change with the development of neoplasia. However, some studies revealed that in some cancers AMPs can be upand in others downregulated; therefore generalization and comparisons should be avoided as the function of antimicrobial proteins may differ between cancers, cancer cells and anatomic localization of the tumor [2]. Also it was

Correspondence: Dr. Marta Fijałkowska, $2^{\text {nd }}$ Department of Plastic, Reconstructive and Aesthetic Surgery, Institute of Surgery, Lodz, Poland, e-mail: fijalkowska.m@wp.pl

Submitted: 12.12.2020; Accepted: 18.05.2021 
reported that HBDs can behave as tumor suppressors as well as proto-oncogenes $[15,16]$. Systematic delineation of the fate and role of the HBDs in a specific cancer type may lead to innovative ways to use defensins as prospective biomarkers for epithelial derived solid tumors for diagnostic/prognostic purposes and/or in novel therapeutic modalities $[2,17]$.

The aim of this paper is to assess the associations between the presence of basal cell carcinoma and the plasma concentrations of antimicrobial peptides such as cathelicidin and $\beta$-defensins (HBD 1-3).

\section{Material and methods}

The study was divided into two terms. The first round took place between March and May 2019 (70 patients were enrolled; it was a pilot study). The second round was conducted between January and March 2020 (38 patients were incorporated). Additionally this division was also caused by the availability of enzyme-linked immunosorbent assays (ELISA). Finally 108 patients were included in the current research. The study group included 50 patients admitted due to skin lesions suspected of cancer for its surgical excision; all of these patients were non-smokers, free from other malignancies and any autoimmunological diseases; and 58 patients were operated on due to other medical conditions, mainly esthetic ones, and were admitted to undergo a one-day procedure in our clinic. All 50 patients with skin neoplasms had their operation done in local anesthesia; the excised skin tumor was sent for histopathological examination. After 2 weeks the results were obtained: 49 patients were diagnosed with BCC while 1 patient had a different, benign lesion and was enrolled in the healthy control group.

Prior any surgical intervention from every patient a $10 \mathrm{ml}$ blood sample was taken. Immediately after collection, the blood was delivered to the Department of Immunology and Allergy, where the sample was centrifuged to obtain plasma. Then it was frozen and stored at $-80^{\circ} \mathrm{C}$ until analysis. After collecting all 108 samples, the plasma was refrozen and the concentrations of $\beta$-defensins (HBD-1, HBD-2, HBD-3) and cathelicidin were measured using an ELISA kit (EIAab Wuhan Science Co., Ltd).

All patients signed written consent forms prior to enrolment in the study. The project was approved by the ethics review board of Medical University of Lodz (approval no. RNN/364/18/KE), and the study was conducted according to the Declaration of Helsinki principles.

\section{Statistical analysis}

The quantitative data were reported as mean \pm standard deviation (SD) or median/interquartile range, according to a normal distribution status fit. For the qualitative data, frequencies and percentages were calculated. Mean differences between the two groups were compared by Student's $t$-test, while the Mann-Whitney $U$ test was applied for comparisons of median values. Receiver-operating characteristic (ROC) curves were used to assess the optimal cutoff points that discriminate between $\mathrm{BCC}$ and healthy groups. Area under the curve (AUC) with standard error (SE) and cathelicidin and HBD 2 level cutoff points using the Youden method were reported, as they provide the best balance between sensitivity and specificity. Multiple logistic regression was applied where presence of the BCC was set as the dependent variable and derived threshold points as well as age were set as independent variables. Odds ratios (ORs) and $95 \%$ confidence intervals $(95 \% \mathrm{CI})$ were calculated. Statistical analyses were conducted using STATISTICA v13.1 (StatSoft Inc., Tulsa, OK, USA). The results with $p<0.05$ were considered as statistically significant.

\section{Results}

In total, 108 patient were included in the analysis (56 women, 52 men). The BCC group consisted of 49 patients with mean age $69.8 \pm 12.3$ and the control group consisted of 59 participants with mean age $62.1 \pm 11.1$. Age was significantly higher in the BCC group than in the control group $(p<0.001)$. In the group of BCC patients 31 of them had the nodular type of cancer, 7 had the superficial type, 7 had the ulcerative type, 2 had the multifocal type, and 2 had the in situ type. The patients had had the cancer for 3 months to 4 years (mean time was 9 months). All the basal cell carcinomas were excised completely.

The average blood concentrations of cathelicidin, HBD1 and HBD2 are presented in Tables 1 and 2. Because the detection rate of HBD1 (12 cases) and HBD3 (1 case) was low, those two defensins were excluded from further analyses. There were no statistically significant differences in concentrations of particular defensins between sexes.

The median serum concentration of cathelicidin was almost 3 times higher and the median concentration of

Table 1. Comparison of measured parameters between genders

\begin{tabular}{|c|c|c|c|c|c|c|c|c|c|c|c|}
\hline \multirow[t]{2}{*}{ Parameter } & \multicolumn{5}{|c|}{ Men } & \multicolumn{5}{|c|}{ Women } & \multirow[t]{2}{*}{$P$} \\
\hline & $n$ & Median & Q1; Q3 & Min. & Max. & $n$ & Median & Q1; Q3 & Min. & Max. & \\
\hline Cathelicidin (pg/ml) & 51 & 815.4 & $547.5 ; 2296.4$ & 208.3 & 6355.4 & 56 & 717.7 & $464.2 ; 1981.5$ & 168.1 & 6778.9 & 0.34 \\
\hline HBD 1 (ng/ml) & 4 & 55.2 & $49.9 ; 91.1$ & 45.2 & 125.6 & 8 & 47.0 & $38.4 ; 63.9$ & 34.8 & 87.8 & - \\
\hline HBD 2 (ng/ml) & 50 & 0.67 & $0.41 ; 4.93$ & 0.17 & 8.37 & 50 & 0.80 & $0.43 ; 4.35$ & 0.16 & 8.87 & 0.82 \\
\hline
\end{tabular}

HBD - human $\beta$-defensin, Q1, Q3 - quartiles 
Table 2. Comparison of measured parameters between healthy group and basal cell carcinoma (BCC) group

\begin{tabular}{|c|c|c|c|c|c|c|c|c|c|c|c|}
\hline \multirow[t]{2}{*}{ Parameter } & \multicolumn{5}{|c|}{ Control group } & \multicolumn{5}{|c|}{ BCC group } & \multirow[t]{2}{*}{$P$} \\
\hline & $n$ & Median & Q1; Q3 & Min. & Max. & $n$ & Median & Q1; Q3 & Min. & Max. & \\
\hline Cathelicidin (pg/ml) & 58 & 613.4 & $443.1 ; 938.1$ & 185.7 & 6355.4 & 49 & 1702.9 & $615.9 ; 2617.8$ & 168.1 & 6778.9 & $<0.001$ \\
\hline HBD 1 (ng/ml) & 7 & 52.1 & $36.9 ; 59.0$ & 34.8 & 125.6 & 5 & 53.8 & $41.8 ; 68.8$ & 39.8 & 87.8 & - \\
\hline HBD $2(\mathrm{ng} / \mathrm{ml})$ & 53 & 0.48 & $0.31 ; 0.83$ & 0.16 & 8.06 & 47 & 3.12 & $0.62 ; 5.69$ & 0.18 & 8.89 & $<0.001$ \\
\hline
\end{tabular}

BCC-basal cell carcinoma, HBD-human $\beta$-defensin, Q1, Q3-quartiles

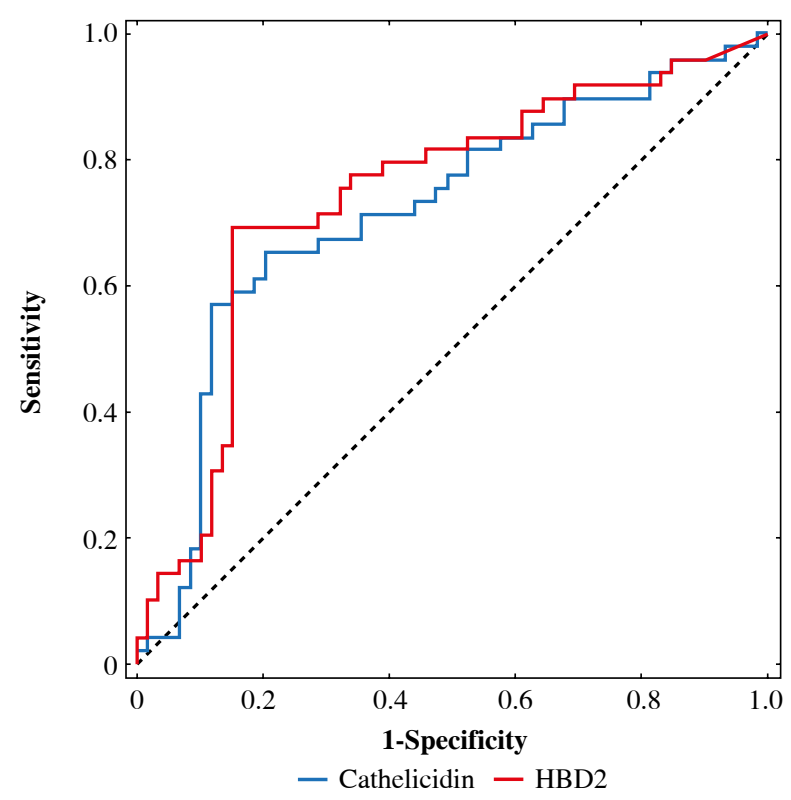

Fig. 1. ROC curves for predicting basal cell carcinoma (BCC) obtained for cathelicidin and HBD-2 for predicting $\mathrm{BCC}$

HBD-2 more than 6 times higher in BCC group than the control group (both $p<0.001$ ).

The ROC curve was derived to assess performance of measurement of both defensins to differentiate between the BCC and control group (Fig. 1). It revealed that AUC for cathelicidin was $0.719 \pm 0.05$ and AUC for HBD-2 was $0.742 \pm 0.05$. There was no statistically significant difference between those values $(p=0.44)$. Graphs of specificity and sensitivity were generated and appropriate thresholds were found using the Youden method (Fig. 2). It was found that the best balance between sensitivity and specificity for cathelicidin to differentiate between BCC and controls was the threshold set at $\sim 1520 \mathrm{pg} / \mathrm{ml}$. Sensitivity and specificity were 0.571 and 0.881 respectively. Further-

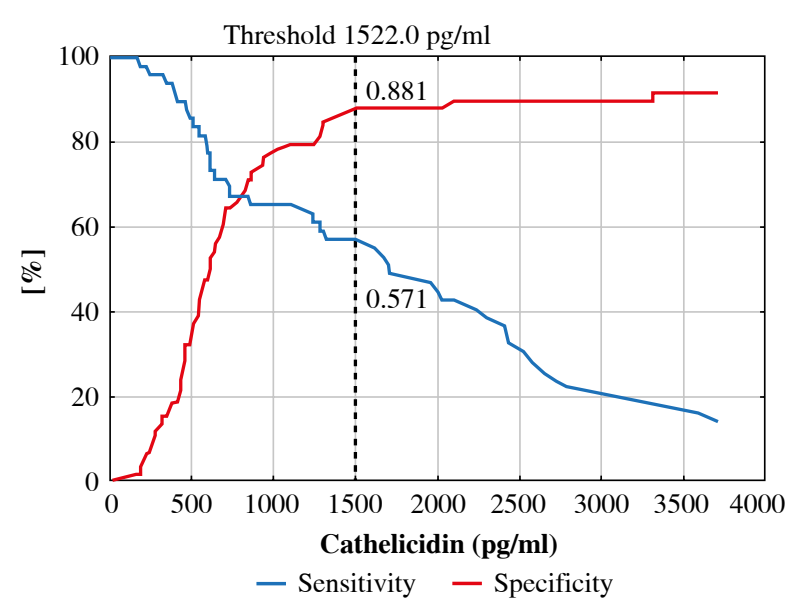

Fig. 2. ROC analysis for cathelicidin concentration with proposed threshold value for predicting basal cell carcinoma (BCC)

more, the threshold for HBD-2 was set as $\sim 1.266 \mathrm{ng} / \mathrm{ml}$ and it was characterized by sensitivity of 0.694 and specificity 0.847 . The remaining indexes of derived threshold are presented in Table 3.

The logistic regression model revealed in univariate analysis that patients who had a detected cathelicidin level above $\sim 1500 \mathrm{pg} / \mathrm{ml}$ had $9.9 \times$ higher likelihood of having BCC identified in the histopathology in comparison with the control group $(\mathrm{OR}=9.9,95 \% \mathrm{CI}: 3.7-26.4, p<0.001)$ (Table 4). In patients who had a HBD-2 level above $\sim 1.2 \mathrm{ng} / \mathrm{ml}$ the OR of having BCC identified in the histopathology was 12.6 (95\% CI: 4.9-32.4, $p<0.001$ ). In the multiple logistic regression model only age was taken into consideration, as no differences were found between sexes in measured parameters. The analysis showed that age of the patients did not affect OR for cathelicidin (OR $=10.2,95 \%$ CI: 3.6-28.9, $p=0.004)$, but increased this ratio for HBD2 (OR $=18.9$, 95\% CI: 6.2-57.9, $p=0.001)$.

Table 3. Comparison of measured parameters between healthy group and basal cell carcinoma (BCC) group

\begin{tabular}{lcccccccc}
\hline Parameter & Threshold & Sensitivity & Specificity & ACC & PPV & NPV & LR(+) & LR(-) \\
\hline Cathelicidin $(\mathrm{pg} / \mathrm{ml})$ & 1522.0 & 0.571 & 0.881 & 0.741 & 0.800 & 0.712 & 4.816 & 0.486 \\
\hline HBD 2 $(\mathrm{ng} / \mathrm{ml})$ & 1.266 & 0.694 & 0.847 & 0.778 & 0.791 & 0.769 & 4.549 & 0.361 \\
\hline
\end{tabular}

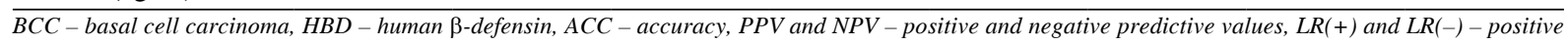
and negative likelihood ratio 
Table 4. Univariate and multivariate logistic regression where detected basal cell carcinoma (BCC) was set as dependent variable

\begin{tabular}{|c|c|c|c|c|c|c|c|c|}
\hline \multirow[t]{2}{*}{ Parameter } & \multicolumn{4}{|c|}{ Univariate } & \multicolumn{4}{|c|}{ Multivariate (+ age) } \\
\hline & OR & $-95 \% \mathrm{CI}$ & $+95 \% \mathrm{CI}$ & $P$ & OR & $-95 \% \mathrm{CI}$ & $+95 \% \mathrm{CI}$ & $\boldsymbol{P}$ \\
\hline Cathelicidin Threshold $1522.0 \mathrm{pg} / \mathrm{ml}$ & 9.9 & 3.7 & 26.4 & $<0.001$ & 10.2 & 3.6 & 28.9 & 0.004 \\
\hline HBD 2 Threshold $1.266 \mathrm{ng} / \mathrm{ml}$ & 12.6 & 4.9 & 32.4 & $<0.001$ & 18.9 & 6.2 & 57.9 & 0.001 \\
\hline
\end{tabular}

\section{Discussion}

In the early 2000 s it became obvious that defensins may play a role in the regulation of carcinogenesis when several studies described altered expression of $\beta$-defensins in cancers [18]. Arimura et al. discovered that in patients with lung cancer concentrations of $\beta$-defensins in serum were elevated [5]. Shi et al. reported that overexpression of HBD2 promotes growth and invasion during esophageal carcinogenesis [4]. Also the authors added that the lower the expression of HBD2 is, the better is the response to the treatment agent. Thus defensin could serve as a biomarker not only to predict the growth of cancer but also to monitor the efficacy of therapy [4]. Wang et al. studied the role of cathelicidin in the development of skin squamous cell carcinoma. They examined a group of 18 patients with SCC, and they concluded that LL-37 can promote proliferation and invasion of tumor cells in squamous cell carcinoma [11].

As basal cell carcinoma is the most frequent skin cancer worldwide, representing 70-80\% of skin neoplasms, and its incidence is growing [19], it would be worth checking how the concentrations of LL-37 and HBDs are fluctuating in serum of patients with BCC. In the literature we can find reports describing altered concentrations of $\beta$-defensins circulating in blood in patients with diffuse bronchiolitis, urinary tract infection and inflammatory bowel disease [20-22]. Measuring blood levels of proteins is quite easy, and it is usually done using the ELISA method, which is reliable and quite cheap in comparison to performing gene expression. Taking into account the abovementioned facts we decided to measure plasma concentrations of $\beta$-defensins and cathelicidin in patients with BCC in comparison to healthy controls using an ELISA kit.

Our study revealed that in patients with BCC, concentrations of both HBD-2 and cathelicidin were significantly higher in comparison to healthy controls. Additionally, we described the association between particular concentrations of LL-37 and HBD-2 and increased risk of having BCC identified in histopathology examination. This risk was significantly higher when the concentration of cathelicidin was more than $1500 \mathrm{pg} / \mathrm{ml}$ and that of HBD-2 more than $1.2 \mathrm{ng} / \mathrm{ml}$. To our knowledge this is the first report describing in such detail the association between cathelicidin and $\mathrm{BCC}$, and the obtained results are promising enough to continue such a scheme of research.

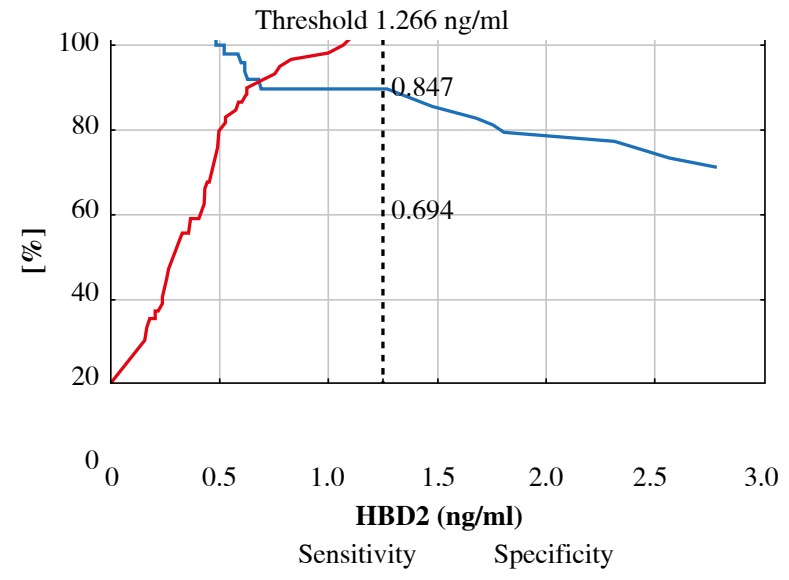

Fig. 3. ROC analysis for HBD-2 concentration with proposed threshold value for predicting basal cell carcinoma (BCC)

This study is not free from limitations. We measured the concentration of defensins and cathelicidin in plasma; further studies can compare those between serum and tissue samples. AMPs are not specific markers only for skin, but in our group we chose patients only with skin neoplasm and no other malignancies. Also it should be remembered that $\mathrm{BCC}$ descended from epithelium, and in this meaning HBDs are specific to epithelial derived solid tumors. Nevertheless, the multiple functions of AMPs place them as important cellular regulators and their proper expression is essential for appropriate function of the immune system. Any imbalance in this network can be a trigger for DNA mutations and carcinogenesis. Further investigations concerning AMPs are necessary to fully realize their potential in diagnosis and therapy in different cancers which have become more and more common. That is why a validation study is planned in the future to multiply and confirm the obtained results.

\section{Conclusions}

Elevated concentrations of cathelicidin and $\beta$-defensin 2 are associated with the presence of basal cell carcinoma. Additionally, the specificity of cathelicidin and $\beta$-defen$\sin 2$ in detecting basal cell carcinoma is high. It can be concluded that cathelicidin and $\beta$-defensin 2 are important 
AMPs playing a role in the genesis of basal cell carcinoma. There is a potential for cathelicidin and HBD-2 to be future markers of skin cancer. However, it should be remembered that these factors are not specific only to this condition and further studies are needed.

\section{The authors declare no conflict of interest.}

\section{References}

1. Kenshi Y, Richard LG (2008): Antimicrobial peptides in human skin disease. Eur J Dermatol 18: 11-21.

2. Ghosh SK, McCormick TS, Weinberg A (2019): Human beta defensins and cancer: contradictions and common ground. Front Oncol 2019; 9: 341.

3. Ritonja A, Kopitar M, Jerala R, Turk V (1989): Primary structure of a new cysteine proteinase inhibitor from pig leucocytes. FEBS Lett 255: 211-214.

4. Shi N, Jin F, Zhang X, et al. (2014): Overexpression of human $\beta$-defensin 2 promotes growth and invasion during esophageal carcinogenesis. Oncotarget 5: 11333-11344.

5. Arimura Y, Ashitani J, Yanagi S, et al. (2004): Elevated serum beta-defensins concentrations in patients with lung cancer. Anticancer Res 24: 4051-4058.

6. Shestakova T, Zhuravel E, Bolgova L, et al. (2008): Expression of human beta-defensins-1, 2 and 4 mRNA in human lung tumor tissue: a pilot study. Exp Oncol 30: 153-156.

7. Markeeva N, Lysovskiy I, Zhuravel E, et al. (2005): Involvement of human beta-defensin-2 in proliferation of transformed cells of human cervix. Exp Oncol 27: 308-313.

8. Gambichler T, Skrygan M, Huyn J, et al. (2006): Pattern of mRNA expression of beta-defensins in basal cell carcinoma. BMC Cancer 6: 163.

9. Scola N, Gambichler T, Saklaoui H, et al. (2012): The expression of antimicrobial peptides is significantly altered in cutaneous squamous cell carcinoma and precursor lesions. Br J Dermatol 167: 591-597.

10. Semlali A, Al Amri A, Azzi A, et al. (2015): Expression and new exon mutations of the human Beta defensins and their association on colon cancer development. PLoS One 10: e0126868.

11. Wang W, Jia J, Li C, et al. (2016): Antimicrobial peptide LL-37 promotes the proliferation and invasion of skin squamous cell carcinoma by upregulating DNA-binding protein A. Oncol Lett 12: 1745-1752.

12. Choi KY, Napper S, Mookherjee N (2014): Human cathelicidin LL-37 and its derivative IG-19 regulate interleukin-32induced inflammation. Immunology 143: 68-80.

13. Wu WK, Wang G, Coffelt SB, et al. (2010): Emerging roles of the host defense peptide LL-37 in human cancer and its potential therapeutic applications. Int J Cancer 127: 1741-1747.

14. Coffelt SB, Tomchuck SL, Zwezdaryk KJ, et al. (2009): Leucine leucine-37 uses formyl peptide receptor-like 1 to activate signal transduction pathways, stimulate oncogenic gene expression, and enhance the invasiveness of ovarian cancer cells. Mol Cancer Res 7: 907-915.

15. Han Q, Wang R, Sun C, et al. (2014): Human beta-defensin-1 suppresses tumor migration and invasion and is an independent predictor for survival of oral squamous cell carcinoma patients. PLoS One 9: e91867.
16. Das Gupta T, Nweze EI, Yue H, et al. (2016): Human papillomavirus oncogenic E6 protein regulates human $\beta$-defensin 3 (hBD3) expression via the tumor suppressor protein p53. Oncotarget 7: 27430-27444.

17. Weinberg A, Jin G, Sieg S, McCormick TS (2012): The Yin and Yang of human beta-defensins in health and disease. Front Immunol 2012; 3: 294.

18. Semple F, Dorin JR (2012): $\beta$-defensins: multifunctional modulators of infection, inflammation and more? J Innate Immun 4: 337-348.

19. Stoddard M, Lyons A, Moy R (2018): Skin cancer prevention: a review of current oral options complementary to sunscreens. J Drugs Dermatol 17: 1266-1271.

20. von Haussen J, Koczulla R, Shaykhiev R, et al. (2008): The host defence peptide LL-37/hCAP-18 is a growth factor for lung cancer cells. Lung Cancer 59: 12-23.

21. Hiratsuka T, Mukae H, Iiboshi H, et al. (2003): Increased concentrations of human beta-defensins in plasma and bronchoalveolar lavage fluid of patients with diffuse panbronchiolitis. Thorax 58: 425-430.

22. Hiratsuka T, Nakazato M, Ihi T, et al. (2000): Structural analysis of human beta-defensin-1 and its significance in urinary tract infection. Nephron 85: 34-40. 